\title{
Association between 53 protein phosphorylated at serine 20 expression and ovarian carcinoma stem cells phenotype: correlation with clinicopathological parameters of ovarian cancer
}

\author{
J. BAR ${ }^{1, \star}$, P. GRELEWSKI ${ }^{1}$, I. DESZCZ ${ }^{1}$, L. NOGA ${ }^{2}$, L. HIRNLE ${ }^{3}$, A. LIS-NAWARA ${ }^{1}$ \\ ${ }^{1}$ Department of Immunopathology and Molecular Biology, Medical University, Wroclaw, Poland; ${ }^{2}$ Department of Pathophysiology, Medical \\ University, Wroclaw, Poland; ${ }^{3}$ First Department of Gynecology, Medical University, Wroclaw, Poland \\ ${ }^{*}$ Correspondence: julia.bar@umed.wroc.pl
}

Received October 12, 2018 / Accepted February 27, 2019

\begin{abstract}
Biological behavior of ovarian carcinomas might be the result of cellular diversity existing in tumor tissue, which consists of differentiated and undifferentiated cells showing stem cells biological properties and function. We examined correlation between p53 protein phosphorylated at serine 20 (p-p53(Ser20)) and CD133, SOX2, Notch1 expression, in order to reveal p-p53(Ser20) stemness function in ovarian cancer. p-p53(Ser20), CD133, Notch1, SOX2 expression was analyzed on 104 ovarian carcinomas using immunohistochemical staining. The positive correlation between $\mathrm{p} 53$ and $\mathrm{p}-\mathrm{p} 53(\mathrm{Ser} 20)(\mathrm{p}=0.02)$, p53 and SOX2 (p=0.02), p-p53(Ser20) and Notch1 ( p=0.03), p-p53(Ser20) and CD133 (p=0.01) expression was observed in ovarian carcinomas. The parallel expression of p-p53(Ser20)/CD133, p-p53(Ser20)/Notch1 reflecting co-expression of these proteins in single carcinoma cell, and p-p53(Ser20)/SOX2 expression was associated with advanced stage and p-p53(Ser20)/ Notch1, p53/SOX2, p-p53(Ser20)/SOX2 parallel expression correlated with high tumor grade. The correlation between p-p53(Ser20) and CD133, Notch1, SOX2 expression and clinical parameters indicate, that malignancy and biological behavior of ovarian carcinomas depend on interaction between p-p53(Ser20) and carcinoma stem cells biomarkers expression.
\end{abstract}

Key words: ovarian carcinoma, 553 phosphorylation, CSCs biomarkers expression

Growing evidence reveals that the limited effectiveness of therapy in patients with ovarian cancer might be the result of morphological and biological heterogeneity of ovarian cancer [1-4]. It was noticed, that ovarian tumors contain two different fractions of tumor cells; one of them is a differentiated tumor cells, the second one appears in an inappropriately differentiated stage described as a dedifferentiated tumor cells which might cause the chemoresistance of ovarian cancer [1,3-4]. This subpopulation of ovarian cancer cells often shows properties similar to normal adult stem cells with the innate capacity to self-renew $[1,4]$. These cells also possess unique features like the ability to initiate the growth and maintenance of tumor heterogeneity and were described as ovarian carcinoma stem cells (CSCs) $[1,4]$. CSCs express specific stem cell markers (e.g. CD133, CD117, CD44, CD24), transcriptional factors (SOX2, OCT4, NANOG), receptors of signaling pathways (Notch, WNT, Hedgehog), which are involved in tumor cell activation and growth [1, 4-7]. Some authors suggested that alterations in oncogenes and suppressor genes, which are observed in ovarian differentiated tumor cells, might occur in CSCs [8-10]. Recently, it was found that p53 has a great impact on processes such as cellular differentiation, self-renewal ensuring a balance between genome stability and plasticity in normal adult stem cells [11-13]. There are data, which show that the activation of p53 inhibits the core transcription factor Nanog, thereby promoting stem cell differentiation in response to DNA and also reduces pluripotent stem cells generation efficiency [13, 14]. Some studies have shown that aberration of p53 expression can promote CSCs initiation and proliferation [5]. There is study, which suggest that p53 functional abnormalities found in mesenchymal stem cells (MSCs) promotes osteosarcoma development [15]. Similarly, p53 abnormalities found in mammary, hematopoietic and neural stem cells could generate aberrant stem cells and promote tumor formation $[11,13]$. It was found, that the activation of p53 alone or in association with other factors is capable of inhibiting cancer via inhibition of stem cell-related mechanisms [13-15]. p53 
protein plays the suppressive function when it is stabilized through posttranslational modification via phosphorylation, acetylation, methylation and ubiquitination [11-14]. Some authors revealed that active forms of $\mathrm{p} 53$ protein are able to inhibit transcription factors Nanog, OCT3/4, Sox 2 which are expressed individually or collectively in many cancer cell types $[11,13,14]$. It has also been demonstrated that active p53 protein expressions in stem cells have been associated with Wnt/ $\beta$ catenin signaling pathways, Sonic Hedgehog (Shh), BIM-1, Notch and PTEN pathways that provide balance of self-renewal and differentiation through niche signaling $[11,13]$. The function of $\mathrm{p} 53$ protein phosphorylation in adult or embryonic stem cells is weakly described [13, 14]. It was found that after DNA damage in embryonic stem cells, p53 binds to the Nanog promoter region and suppresses Nanog expression through serine 213 or serine 315 p53 phosphorylation and promotes stem cells differentiation [11-13]. In mice model has been found, that p53 protein phosphorylated at serine 23 and threonine (Thr) 21 (equivalent to human Thr18, Ser20), shows depletion of multiple stem cell populations, mainly through p53-mediated expression of PUMA [16]. Lately, Kamińska et al. analyzed p53 phosphorylation at serine 15 and 20 in ovarian carcinoma cell lines and cells isolated form ascitic fluid of patients with ovarian cancer and their results revealed that sensitivity to chemotherapy used depend on p53 protein phosphorylation status [17]. Moreover, it was found that p53 protein phosphorylated at Ser 15 and Ser20 determined the apoptotic activity of ovarian carcinoma cells after chemotherapy [17]. The mechanisms by which wild-type p53 or mutant p53 influence stemness in non-malignant stem cells and CSCs are poorly understood [13]. Likewise, the role of p53 wildtype protein or mutant protein isoforms phosphorylation in cancer stem cells biology is unclear. Several authors suggest that active mutant $\mathrm{p} 53$ protein showed oncogenic properties that are independent of wild type of p53 [11, 13, 14]. There are no data on whether phosphorylation of mutant p53 is necessary to initiate tumor formation by promoting the generation and expansion of pluripotent cells $[13,14]$. Up to now, there are no studies on p53 phosphorylation at serine 20 in ovarian carcinoma cells demonstrating a cancer stem-like phenotype. Nothing is known about the interaction between p53 protein phosphorylation at serine 20 (p-p53(Ser20) and stem cells biomarkers expression in relation to biological and clinical features of ovarian cancers.

The aim of this study was to estimate p-p53(Ser20) expression in relation to cancer stem cells biomarkers e.g. CD133, SOX2, Notch1 in order to reveal p-p53(Ser20) protein stemness function in ovarian cancer.

\section{Patients and methods}

Patients. One hundred and four patients with primary ovarian carcinoma before therapy were entered in this study between May 2008 and July 2016. Formalin-fixed, paraffin- embedded tumor tissues from patients with ovarian carcinoma were obtained from the First Gynecologic Clinic of Medical University, Wroclaw, Poland. Malignant tumors were staged according to the International Federation of Gynecology and Obstetrics (FIGO) criteria [18]. Nine tumors were in stage I, thirty in II, fifty were in III, fifteen in IV FIGO stage.

Tissue specimens. Ovarian tumor specimens were histologically verified to confirm the histological type and grade of tumor according to earlier established criteria [19]. The study consists of 35 serous, 39 endometrioid, 18 undifferentiated and 12 mucinous ovarian carcinomas. Thirty-two tumors were well (G1), 35 moderately (G2) and 37 poorly (G3) differentiated.

Cell lines. A primary ovarian cell line OvBH-1 with p53 mutation in exon 6 codon 224 change from GAG to GA $\underline{A}$ (patent PL189880) and cell line SW626 derived from an ovarian metastasis of a primary adenocarcinoma of the colon with p53 mutation in exon 8 codon 262 change from GGT to GTT (ACCC, USA) were used in this study. Cell lines were cultured in Dulbecco's modified Eagle's medium (DMEM) with $10 \%$ fetal calf serum (Gibco, Karlsruhe, Germany) and supplemented with antibiotics (penicillin/streptomycin; Sigma-Aldrich, Poznan, Poland). The cells were maintained in a humidified atmosphere at $37^{\circ} \mathrm{C}$ and $5 \% \mathrm{CO}_{2}$. When cells reached confluence, they were removed by trypsinization (Trypsin 0.25\%; Sigma-Aldrich, Poznan, Poland) and used for further experiments. For immunohistochemical staining the cells specimens were fixed $10 \mathrm{~min}$ in cold acetone and dry for $20 \mathrm{~min}$ at room temperature.

Antibodies. Immunohistochemical staining of the p53 protein was performed with the following antibodies: mouse monoclonal antibody DO-7 (clone 7) reacts with both wild- and mutant forms of unphosphorylated human p53 protein, recognizing an epitope between amino acids 20 and 25 (Novocastra, Newcastle, UK). Rabbit polyclonal antibody phospho-specific p53 (Ser20) detects endogenous levels of the $\mathrm{p} 53$ protein phosphorylation at serine 20 (\#9287, Cell Signaling Technology, Boston, USA). Rabbit polyclonal anti-CD133 antibody (orb9913, Biorbyt, Cowley, UK), rabbit polyclonal antibody anti-SOX2 transcriptional factor (orb11398, Biorbyt, UK), rabbit monoclonal antibody anti-Notch1 (D1E11) receptor detects intracellular epitopes between 2400-2 500 amino acids of human Notch1 (\#3608, Cell Signaling Technology, Boston, USA).

Immunohistochemistry. Immunohistochemical staining for analyzed proteins was performed on paraffin-embedded ovarian carcinomas tissue and on cytospin preparations of OvBH-1 and SW626 cells using the Universal Dako REAL EnVision Detection System, Peroxidase/DAB+, Rabbit/ Mouse (Dako, Copenhagen, Denmark) and primary antibodies: anti-p53 protein, anti-p-p53(Ser20), antiNotch1, anti-CD133, anti-SOX2. Four- $\mu \mathrm{m}$ sections from one selected block from each ovarian tumor were deparaffinized and boiled $2 \times 5 \mathrm{~min}$ in a citrate buffer $(\mathrm{pH}=6.0)$ at $800 \mathrm{~W}$ 
Table 1. The association between expression of total p53 protein, p53, p-p53(Ser20), stem cell markers and clinico-pathological factors of ovarian carcinomas.

\begin{tabular}{|c|c|c|c|c|c|c|c|c|c|c|c|}
\hline \multirow{3}{*}{ Parameters } & \multicolumn{11}{|c|}{ Immunoreactivity } \\
\hline & \multirow{2}{*}{$\begin{array}{c}\text { No cases } \\
\mathbf{n}\end{array}$} & \multicolumn{2}{|c|}{ p53 } & \multicolumn{2}{|c|}{ p-p53(Ser20) } & \multicolumn{2}{|c|}{ CD133 } & \multicolumn{2}{|c|}{ Notch1 } & \multicolumn{2}{|c|}{ SOX2 } \\
\hline & & n [\%] & p-value & n [\%] & p-value & n [\%] & p-value & n [\%] & p-value & n [\%] & p-value \\
\hline \multicolumn{12}{|c|}{ Histological subtype } \\
\hline \multicolumn{12}{|c|}{ of ovarian cancers } \\
\hline serous & 35 & $17(48.5)$ & & $19(54.2)$ & & $12(34.2)$ & & $12(34.2)$ & & $30(85.7)$ & \\
\hline non-serous & 69 & $25(36.2)$ & NS & $27(39.1)$ & NS & $28(40.5)$ & NS & $22(31.8)$ & NS & $53(76.8)$ & NS \\
\hline \multicolumn{12}{|l|}{ FIGO stages } \\
\hline I/II & 39 & $15(38.4)$ & & $10(25.6)$ & & $12(30.7)$ & & $9(23.0)$ & & $29(74.3)$ & \\
\hline III/IV & 65 & $27(41.5)$ & NS & $36(55.3)$ & 0.003 & $28(43.0)$ & NS & $25(38.4)$ & NS & $54(83.0)$ & NS \\
\hline \multicolumn{12}{|l|}{ Tumor grade } \\
\hline $\mathrm{G} 1 / \mathrm{G} 2$ & 67 & $22(32.8)$ & & $22(32.8)$ & & $25(37.3)$ & & $17(25.3)$ & & $50(74.6)$ & \\
\hline G3 & 37 & $20(54.0)$ & 0.035 & $24(64.8)$ & 0.016 & $15(40.5)$ & NS & $17(45.9)$ & 0.032 & $33(89.1)$ & 0.045 \\
\hline
\end{tabular}

$\mathrm{n}$ - number of positive cases. NS - no statically significant.

in a microwave. After the microwave treatment, the tissue sections were slowly cooled for 30 minutes. Nonspecific tissue and endogenous peroxidase reactivity were blocked with Dako REAL Peroxidase Blocking Solution (Dako, Copenhagen, Denmark). Tissue specimens were incubated with primary antibodies overnight at $4^{\circ} \mathrm{C}$. Cells specimens were incubated with primary antibodies $60 \mathrm{~min}$ at room temperature. After washing the tissue and cells specimens with $0.1 \mathrm{M}$ Tris-buffer, $\mathrm{pH}=7.4$ (TBS), they were incubated with Dako REAL EnVision/HRP, Rabbit/Mouse (Dako, Copenhagen, Denmark) for $30 \mathrm{~min}$ at room temperature. After washing with TBS the antigen-antibody reaction was visualized by DAB (3,3-diaminobenzidine) (Dako, Denmark) as a chromogen. Sections were counterstained with hematoxylin and mounted. The incubation buffer (TBS) without primary antibodies was used as a negative control. Positive controls for each antibody were performed according the manufacturers' recommendation. In selected representative cases of ovarian carcinomas as well as in cell lines double staining for p-p53(Ser20) and CD133, or Notch1 was performed using EnVision DuoFLEX System according to the manufactures' procedure (Dako, Copenhagen, Denmark).

Interpretation of immunohistochemical staining. Assessment of analyzed proteins expression in tumor tissue and cell specimens was scored semiquantitatively, taking into account the intensity of immunostaining and number of tumor cells showing immunoreactivity for analyzed proteins. The number of tumor cells exhibiting staining for p53, p-p53(Ser20), and SOX2 antibodies were assessed by counting 1000 cells in randomly selected 10-15 high power fields. CD133 and Notch1 expression was analyzed by determining cytoplasmic/membrane immunostaining based on the intensity of immunostaining and the percentage of stained tumor tissue area. In cytospin preparation, the percentage of positive cells for p53, p-p53(Ser20), SOX2, CD133, Notch1 proteins expression was determined by counting 1000 cells in randomly selected areas. The cases were scored as negative for p53, p-p53(Ser20) and stem cells markers (CD133, Notch1, SOX2) when there was no immunostaining or variable weak positivity ( $<10 \%$ of tumor cells).

Statistical analysis. Correlations between p53 protein, p-p53(Ser20), CD133, Notch1, SOX2 expression and clinicopathological parameters in ovarian carcinomas were statistically studied by the Chi-square test. Associations between p53 protein, p-p53(Ser20), and CD133, Notch1, SOX2 expression were analyzed by the Spearman rank correlation. Associations between the parallel expression of the analyzed proteins and FIGO stages, tumor grade, histological type were performed using the Chi-square test. For all statistical analyses, p53, p-p53(Ser20), CD133, Notch1, SOX2 expression was divided into two groups: negative or limited to $10 \%$ of positive tumor cells versus $>10-100 \%$ of positive cells. Differences were considered significant when $\mathrm{p}<0.05$.

\section{Results}

Immunoreactivity for p53, p-p53(Ser20) was detected in $40.3 \%, 44.2 \%$ of ovarian carcinomas, respectively. Whereas, p53 and p-p53(Ser20) expression was observed in 70\% and $30 \%$ of OvBH-1 cells and in $30 \%$ and $20 \%$ of SW626 cells, respectively. CD133, Notch1, and SOX2 expression was found in $38.4 \%, 32.6 \%$, and $74.0 \%$ of ovarian carcinomas, respectively. Immunoreactivity for CD133, Notch1, and SOX2 was observed in $30 \%, 20 \%$, and $30 \%$ of OvBH-1 cells, respectively. Notch1 and SOX2 expression was observed in $20 \%$ and $40 \%$ of SW626 cells respectively. CD133 molecule expression was not found in SW626 cells. Variations in p53, p-p53(Ser20) and in CD133, Notch1, SOX2 expressions were observed not only in the whole group of cases, but also in individual ovarian cancer as well as in cell lines. Immunoreactivity for p53 and p-p53(Ser20) was found in >10 to $90 \%$ of positive cells. Whereas, expression of CD133, Notch1 and SOX2 was observed in $>10$ to $60 \%$ of positive tumor cells. However, the intensity of immunostaining and range of 
positive cells for analyzed biomarkers in ovarian carcinoma tissue was higher in advanced stage and poorly differentiated (Figure 1), than early stage and well differentiated ovarian carcinomas (Figure 2). In OvBH-1 and SW626 cell lines p53, Ser20 and stem cells biomarkers expression showed heterogeneous immunostaining (Figure 3). The association between the analyzed biomarkers and clinicopathological parameters is presented in Table 1. p-p53(Ser20) expression was mainly detected in stages III/IV than in FIGO stage I/II ( $\mathrm{p}=0.003)$. p53, p-p53(Ser20), Notch1 and SOX2 expression was significantly associated with poorly differentiated (G3) ovarian carcinomas (Table 1). The positive correlation between p53 and $p$-p53(Ser20) $(p=0.02)$ was found in ovarian carcinomas. However, $42.8 \%$ cases with nuclear accumulation of p53 (p53-positive) were negative for p-p53(Ser20) expression, but $35.4 \%$ cases without nuclear accumulation of p53
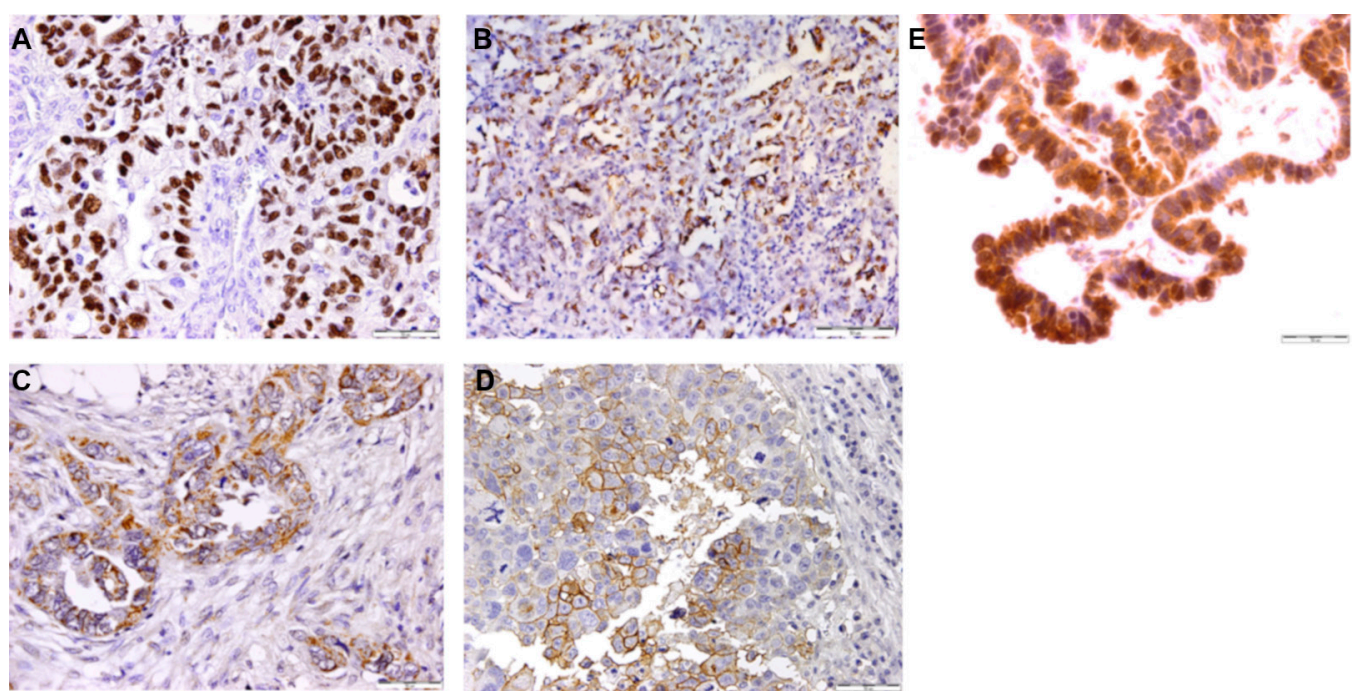

Figure 1. High advanced ovarian carcinomas with p53 protein, p-p53(Ser20), CD133, Notch1, SOX2 expression. Endometrioid ovarian carcinoma FIGO stage III, poorly differentiated (G3) showing strong nuclear accumulation of p53 protein (A), undifferentiated ovarian carcinoma FIGO stage III, expressing p-p53(Ser20) in high percentage of tumor cells (B), serous ovarian carcinoma FIGO III, poorly differentiated (G3) showing cytoplasmic/ membrane CD133 expression limited to small area of tumor tissue (C), endometrioid ovarian carcinoma FIGO stage III moderately differentiated (G2), exhibiting heterogeneous membrane Notch1 expression in few tumor cells (D), serous ovarian carcinoma FIGO stage III moderately differentiated (G2), showing extensive cytoplasmic/nuclear SOX2 expression (E) (EnVision technique). The scale bar $50 \mu \mathrm{m}$.
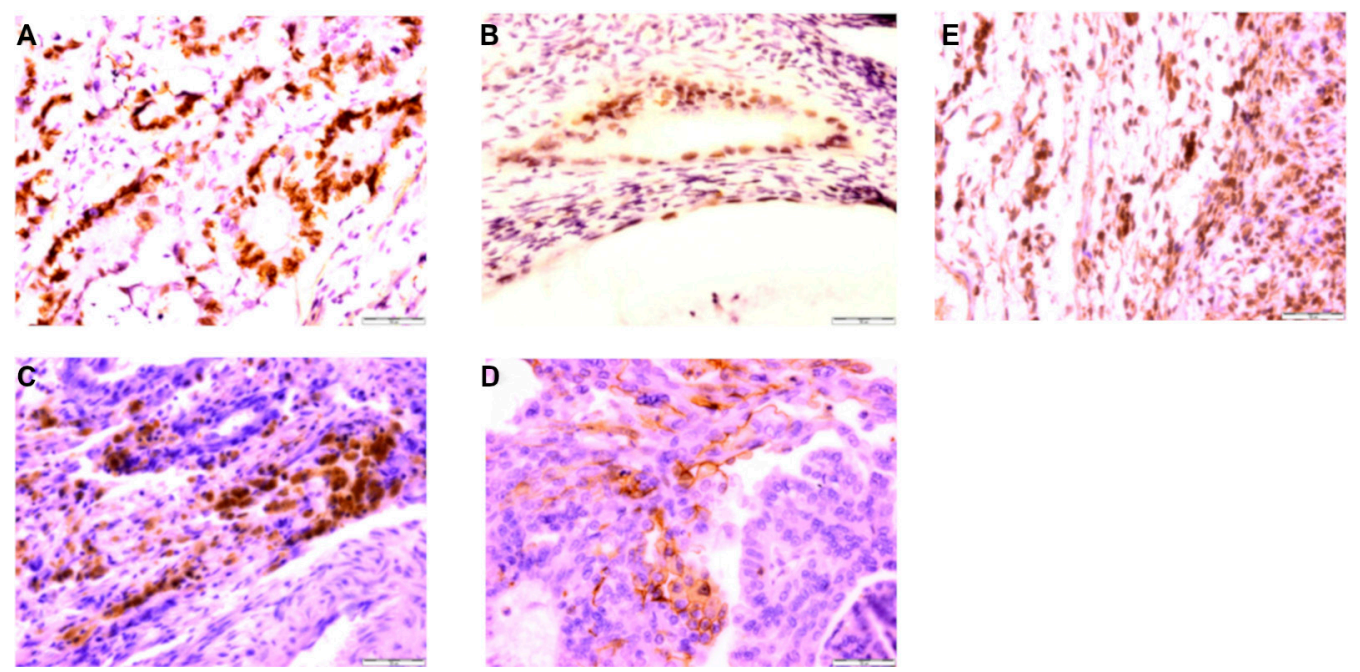

Figure 2. Low advanced ovarian carcinomas with p53 protein, p-p53(Ser20), CD133, Notch1, SOX2 expression. Endometrioid ovarian carcinoma FIGO stage II, well differentiated (G1) heterogeneous pattern of p53 immunoreactivity in tumor tissue (A), mucinous ovarian carcinoma FIGO stage I, well differentiated (G1) weak expression of p-p53(Ser20) limited to a small area of tumor tissue (B), endometrioid ovarian carcinoma FIGO stage II, well differentiated (G1) with focus CD133 expression in tumor tissue (C), serous ovarian carcinoma FIGO stage II, well differentiated (G1) with low expression of Notch1 (D), undifferentiated ovarian carcinoma FIGO stage II heterogeneous pattern of nuclear SOX2 expression is visible (E) (EnVision technique). The scale bar $50 \mu \mathrm{m}$. 
(p53-negative) showed p-p53(Ser20) expression. Association between $\mathrm{p} 53$ and SOX2 (p=0.02), p-p53(Ser20) and Notch1 $(\mathrm{p}=0.03), \mathrm{p}-\mathrm{p} 53($ Ser20) and CD133 $(\mathrm{p}=0.01)$ expression was observed in ovarian carcinomas. The association between analyzed biomarkers was confirmed by double immunohistochemical staining. Heterogeneous pattern of p-p53(Ser20) and CD133, and p-p53(Ser20) and Notch1 expression was observed in selected cases of ovarian carcinoma. CD133 and Notch1 expression was found in p-p53(Ser20) positive and $\mathrm{p}$-p53(Ser20) negative ovarian carcinoma cells. Co-expression of p-p53(Ser20)/CD133 was observed mainly in p53 positive ovarian carcinoma cells (Figure 4A), whereas p-p53(Ser20)/Notch1 co-expression was limited to single or few carcinoma cells (Figure 4B). In cell lines only OvBH-1 cells showed co-expression of p-p53(Ser20)/CD133 (Figure 4C). Ovarian cancer cells showing parallel expression of CD133, Notch1, SOX2 revealed a more frequent expression of $\mathrm{p} 53(\mathrm{p}=0.002)$ and $\mathrm{p}-\mathrm{p} 53(\operatorname{Ser} 20)(\mathrm{p}=0.0001)$. In Figure 5, the association between the parallel expression of analyzed biomarkers and clinicopathological parameters of ovarian carcinomas is presented. The expression of p-p53(Ser20)/ CD133, p-p53(Ser20)/Notch1, p-p53(Ser20)/SOX2 was observed more often in III/IV than in I/II FIGO stage $(\mathrm{p}=0.01$, $\mathrm{p}=0.004, \mathrm{p}=0.001$, respectively) (Figure 5A). $\mathrm{p}-\mathrm{p} 53($ Ser20)/ Notch1, p53/SOX2, p-p53(Ser20)/SOX2 parallel expression was associated with high tumor grade $(p=0.01, p=0.005$, $\mathrm{p}=0.009$, respectively) (Figure $5 \mathrm{~B}$ ). No correlation between parallel of Ser20 and stem cells biomarkers (CD113, Notch1 SOX2) expression and histological type of ovarian carcinoma was observed. Only p53/SOX2 immunophenotype was found in serous ovarian cancer $(\mathrm{p}=0.01)$.

\section{Discussion}

Growing evidence demonstrated that p53 loss could lead to an acquisition of stemness in many solid tumors $[5,11$, 13]. The role of $\mathrm{p} 53$ protein was described in carcinoma stem cells but little is known about p53 protein phosphorylation at serine 20 in CSCs biological behavior [11, 12, 14, 15]. Similarly to earlier published data, we found that p53 nuclear accumulation was associated with worse clinical features of ovarian cancers [20-26]. p53 protein phosphorylation was analyzed very rarely in ovarian carcinomas and there are no data on its expression in ovarian carcinoma stem cells [27, 28]. Observed in current study, expression of $\mathrm{p} 53$ protein phosphorylated at Ser20 in $44.2 \%$ of cases was associated with advanced stage and poorly differentiated ovarian carcinomas. These results suggest that p53 protein phosphorylation in ovarian cancers might have impact on tumor cells biological, morphological features and enhanced tumor growth $[28,29]$. Association between p53 nuclear accumulation and serine 20 expression observed in current paper suggests that $\mathrm{p} 53$ protein phosphorylation might concern not only wild type, but also mutant forms of p53 protein overexpressed frequently in advanced and poorly differenti- ated ovarian carcinomas $[22,25,26]$. This suggestion might be supported by Kaminska et al. [17] results, who found that expression of p53 protein phosphorylated at Ser20 was observed in cell lines of primary ovarian carcinoma and metastasis of a colon adenocarcinoma to ovary with TP53 gene mutation and high p53 protein accumulation. Also Nguyen et al. [29] indicate that most mutant p53 isoforms
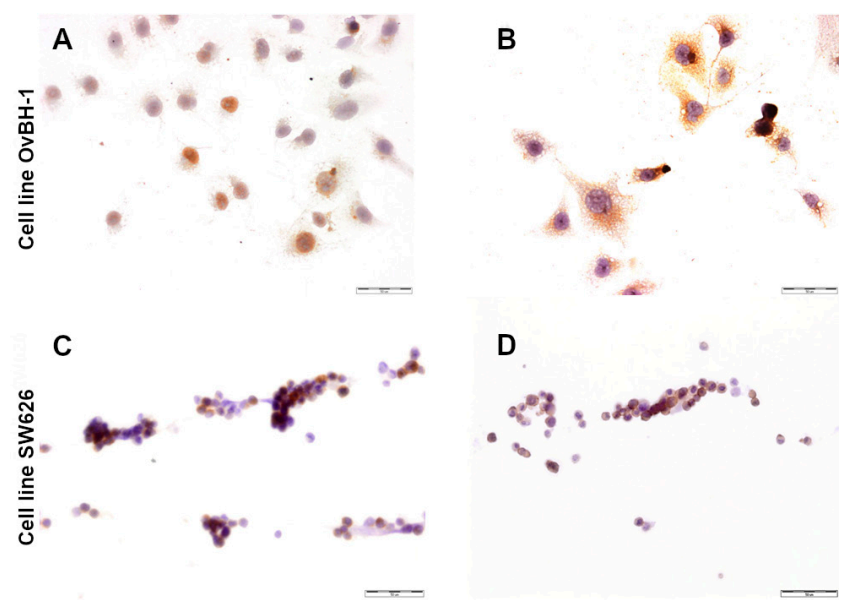

Figure 3. Expression of p-p53(Ser20) and stem cells biomarkers in cell lines: OvBH-1 cell line showed high nuclear p-p53(Ser20) expression (A), limited expression of CD133 molecules in OvBH-1 cells (B), SW626 cells with strong expression of p-p53(Ser20) in high percentage of cells (C), Notch1 expression was detected in large number of SW626 cells (D). (EnVision technique). The scale bar $50 \mu \mathrm{m}$.

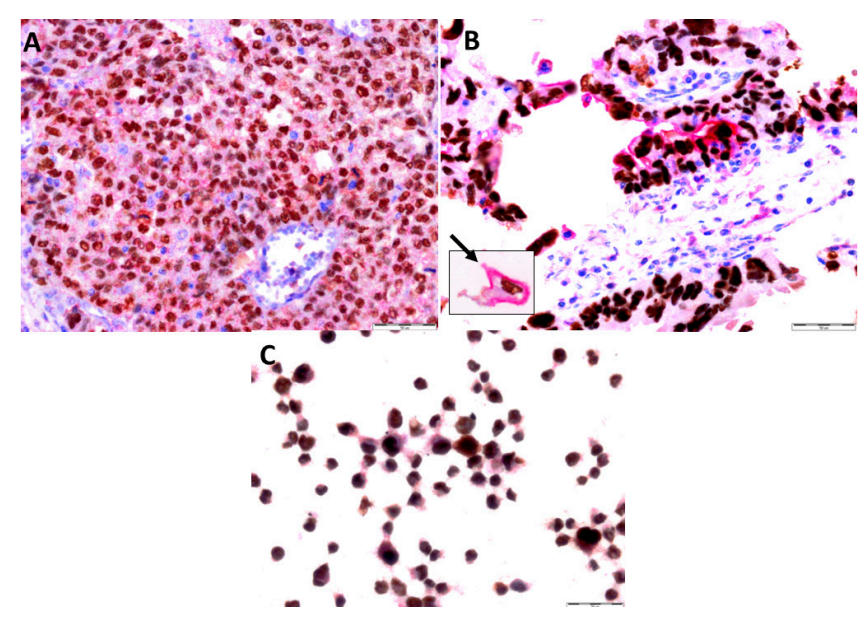

Figure 4. p-p53(Ser20) and CD133, Ser20 and Notch1 double staining in representative cases of ovarian carcinoma and cell line. Serous ovarian carcinoma FIGO stage III, poorly differentiated (G3) with co-expression of p-p53(Ser20) located in the nucleus (brown) and CD133 in the cytoplasm (red immunostaining) observed in moderate percentage of tumor cells (A), endometrioid ovarian carcinoma FIGO stage III, poorly differentiated (G3) showing coexpression of p-p53(Ser20) located in the nucleus (brown) and Notch1 receptor located in the membrane/cytoplasm (red) dominated in malignant tumor cells (marked by arrow) (B). OvBH1 cell line with coexpression of p-p53(Ser20) visible in nucleus (brown) and CD133 expression visible in the cytoplasm $(\mathrm{C})$. EnVision technique and EnVision DuoFLEX System. The scale bar $=50 \mu \mathrm{m}$. 

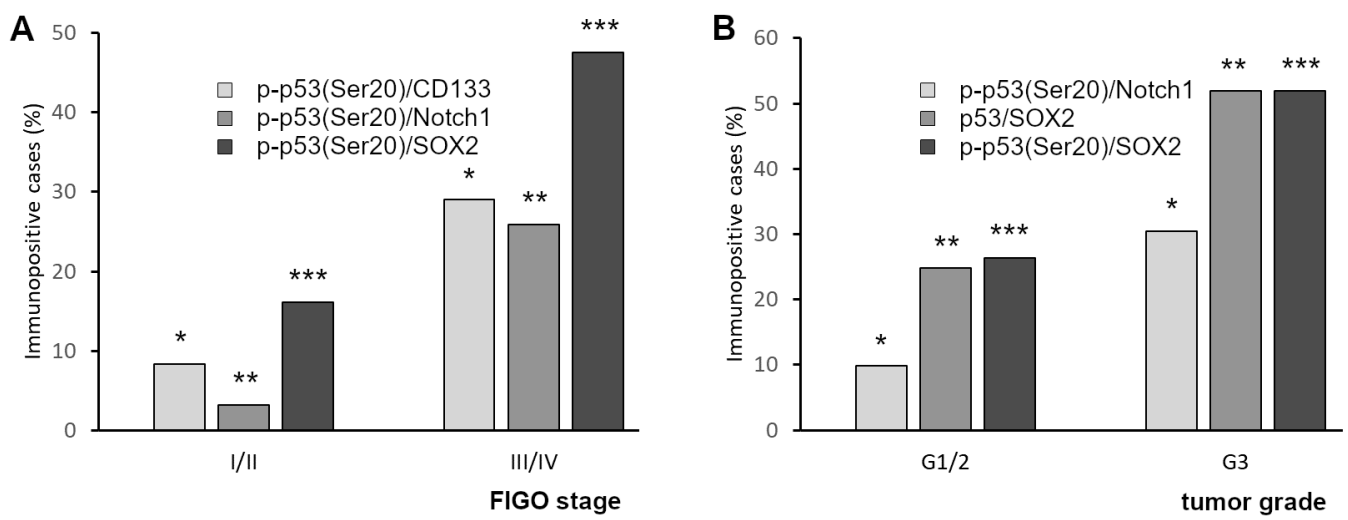

Figure 5. Association between ovarian carcinoma immunophenotype and clinico-pathological parameters. A) Immunophenotypes of p-p53(Ser20)/ CD133, p-p53(Ser20)/Notch1, p-p53(Ser20)/SOX2 was dominated in III/IV in comparison to I/II FIGO stage of ovarian carcinomas (*: p=0.01, $\left.\left.{ }^{* *}: \mathrm{p}=0.004,{ }^{* *}: \mathrm{p}=0.001\right) ; \mathrm{B}\right)$ Immunophenotypes of $\mathrm{p}-\mathrm{p} 53($ Ser20)/Notch1, p53/SOX2 p-p53(Ser20)/SOX2 was associated with high ovarian carcinoma grade $\left({ }^{*}: \mathrm{p}=0.01,{ }^{* *}: \mathrm{p}=0.005,{ }^{* * *}: \mathrm{p}=0.009\right)$.

can be posttranslationaly modified at the same residues as in wild type p53 protein and contribute to a gain of function leading to increased tumor progression. In the literature, there are no data describing Ser20 expression in ovarian carcinoma stem cells. In current study, the stemness features of ovarian carcinoma cells were analyzed by expression of biomarkers occurring on normal adult stem cells e.g. CD133, SOX2, Notch1 [11, 13]. Similarly to published data, the present authors noted that ovarian carcinoma stem cells were identified by CD133 molecules in ovarian tumor tissue [30-33]. However, contrary to the published reports the associations between CD133 expression and clinicopathological parameters of ovarian cancer were not observed in the current study [30,31]. Nevertheless, there are data suggesting that it is still controversial whether CD133 expression correlated with clinicopathological parameters of ovarian carcinomas and had an impact on the aggressive biological behavior of ovarian cancer [32, 33]. Experimental studies revealed that p53 protein might inhibit CD133 expression and modulate the expression of CSCs specific genes such as: Notch1, SOX2 [5, 12, 13, 26]. The role of p53 phosphorylation was described in normal adult stem cells but in CSCs its role has not been established yet $[12,13]$. In the current study, a positive correlation between CD133 and Ser20 expression was observed in ovarian carcinomas. The use of double immunostaining technique allows to reveal the co-expression between p-p53(Ser20) and CD133 molecules in single ovarian carcinoma cell $[34,35]$. The co-expression of p-p53(Ser20) and CD133 molecule was found in ovarian carcinomas with nuclear $\mathrm{p} 53$ protein expression, but also was observed in cases without $\mathrm{p} 53$ protein expression detected by DO-7 antibody. We might consider that p53 protein phosphorylation at serine 20 found in p53-positive and p53-negative ovarian carcinoma cells might concern different forms of $\mathrm{p} 53$ protein $[12,25]$. The presence of $\mathrm{p} 53$ protein phosphorylated at Ser20 in ovarian carcinomas negative for
DO-7 antibody immunostaining may point out that the wild type of p53 protein is low, but is stabilized in response to stress inducing marked posttranslational modification of p53 protein $[12,13]$. According to previous data, parallel p-p53(Ser20)/CD133 expression accompanied by co-expression of these proteins in $\mathrm{p} 53$ negative ovarian cancers indicate that the role of wild-type p53 phosphorylated at Ser20 might be similar to normal adult stem cells and p53 protein phosphorylated at Ser20 can suppress CD133 expression and inhibits tumor growth $[11,12]$. Based on published data, we can suggest that wild-type p53 protein phosphorylated at Ser20 might play such roles by suppression of stemness transcription factors $[12,13,16]$. The interactions between p-p53(Ser20) and CD133 expression in p53-positive ovarian carcinomas are not clear now $[12,13,16]$. However, confirmed by double staining the association between parallel expression of p-p53(Ser20)/CD133 and advanced stage of ovarian cancer indicates that ovarian cancer growth might depend not only on stemness features of tumor cells, but also on p53 protein status phosphorylation $[17,28]$. Contrary to earlier reports, in the present study Notch 1 expression was found in $38.6 \%$ of ovarian cancers $[6,36]$. Similarly to Wang et al. [32], we found that Notch1 expression was associated with poorly differentiated ovarian cancers. This result indicates that Notch1 expression might be the biomarker characterizing undifferentiated ovarian carcinoma cells and might influence ovarian tumor growth $[9,13,32]$. There are no data on parallel Notch1/p-p53(Ser20) expression in ovarian carcinomas. In current study we found that parallel expression of Notch1/p-p53(Ser20) expression was observed as co-expression of these biomarkers but were visible in small number of ovarian carcinoma cells. These results indicate that Notch1 expression might depend on p-p53(Ser20) expression and the role of Notch1 in ovarian carcinomas might be determined by $\mathrm{p} 53$ protein phosphorylation. We postulated that co-expression of Notch1/p-p53(Ser20) might characterize 
subclones of ovarian carcinoma cells, which possess features promoting their selective growth in a tumor mass. There are strong evidences showing correlation between TP53 gene and Notch members in ovarian cancer, which are involved in various aspects of stem cells maintenance, cell differentiation and tumor progression [26]. We found that parallel Notch1/ p-p53(Ser20) expression was accompanied by co-expression of Notch1/p-p53(Ser20) and was correlated with advanced and poorly differentiated ovarian cancers. This observation might be partly compared with Chen et al. [26], who revealed that TP53 status significantly impact the prognostic value of Notch 1 expression in ovarian cancer. The role of SOX2 expression as an important marker, which demonstrates an oncogenic function in epithelial cancer cells by promoting cell proliferation, cell migration and tumorigenic potential was found $[6,13,33]$. In the present study SOX2 expression was observed in high percentage of poorly differentiated ovarian carcinomas [7,37]. This observation suggests that the activity of SOX2 in poorly differentiated ovarian carcinomas might promote their malignancy and tumor growth $[7,11,13,38,39]$. In the current study, an observed association between parallel p53 nuclear accumulation and SOX2 expression in poorly differentiated ovarian carcinomas is in agreement with published studies. These indicate that nuclear accumulation of p53 protein might enhance transcriptional activity of SOX2 in tumor cells and facilitate their function as an oncogene promoting stemness phenotype of ovarian cancer reflecting the biologically aggressive feature of ovarian cancer $[7,11,13,15,38,39]$. Up to now there are no data on association between p-p53(Ser20) and SOX2 expression in ovarian carcinomas. In the next step of our research, we analyzed the association between p53 phosphorylation at Ser20 and SOX2 expression in ovarian carcinomas. We found that in the whole group of analyzed cases the correlation between p-p53(Ser20) and SOX2 was not observed. However, parallel expression of both SOX2 and p-p53(Ser20) was associated with advanced stage and poorly differentiated ovarian cancers. According to published results, we might conclude that regulation of SOX2 transcriptional activity might depend on 553 protein phosphorylated form at serine 20 and has an impact on ovarian carcinoma cells behavior [11-13]. This suggestion might be supported by data, which revealed that wild-type p53 loss has been associated with enhancing the efficiency of somatic cell reprogramming to a pluripotent state and stem-like phenotype in cancer [11, 13, 39]. Revealed in the current paper, association between the parallel expression of p-p53(Ser20)/Notch1, p-p53(Ser20)/ CD133 accompanied by co-expression of these biomarkers and advanced and poorly differentiated ovarian carcinomas indicate that $\mathrm{p} 53$ protein phosphorylation at serine 20 might concern different forms of $\mathrm{p} 53$ protein expressed by ovarian cancer stem cells and might implicate CSCs motility and invasion leading to different biological behavior of ovarian cancer $[11,13,39]$. Found in OvBH-1 cell line with TP53 gene mutation co-expression between p-p53(Ser20) and
CD133 might confirm our hypothesis. On the other hand, some authors suggested that the role of $\mathrm{p} 53$ protein phosphorylation as a pivotal mechanism, which controls expression of transcriptional factors in adult stem cells and prevents uncontrolled de-differentiation of stem cells [11-14]. According to published results, in our opinion this mechanism might be disturbed due to the phosphorylation of different forms of p53 protein [12-14]. Results from this study showed that correlation between p-p53(Ser20) and CD133, Notch1, expression reflects co-expression of these proteins in single ovarian carcinoma cell indicating that p-p53(Ser20) might play stemness role in ovarian carcinomas and cooperation between these markers might have impact on biological behavior of ovarian cancer. Association between p53 phosphorylated at serine 20 and carcinoma stem cells biomarkers suggest that these biomarkers cooperate and network between these proteins might facilitate ovarian tumor growth. Association between parallel p-p53(Ser20)/ Notch1, p-p53(Ser20)/CD133, p-p53(Ser20)/SOX2 expression and advanced stage and high tumor grade indicate that these biomarkers enhancement malignancy of ovarian carcinomas with stem cell phenotype.

Acknowledgments: This study was supported by the grant No.755 from the Medical University Wrocław, Poland.

\section{References}

[1] SHAH MM, LANDEN CHN. Ovarian cancer stem cells: are they real and why are they important? Gynecol Oncol 2014; 132: 483-489. https://doi.org/10.1016/j.ygyno.2013.12.001

[2] NOZAWA-SUZUKI N, NAGASAWA H, OHNISHI K, MORISHIGE K. The inhibitory effect of hypoxic cytotoxin on the expansion of cancer stem cell in ovarian. Biochem Biophys Res Commun 2015; 457: 706-711. https://doi.org/10.1016/j. bbrc.2015.01.053

[3] ROY L, SAMYESUDHAS SJ, CARRASCO M, LI J, JOSEPH $S$ et al. ARID3B increases ovarian tumor burden and is associated with a cancer stem cell signature. Oncotarget 2014; 18: 8355-8366. https://doi.org/10.18632/oncotarget.2247

[4] RICCI F, GUFFANTI F, DAMIA G. Ovarian cancer recurrence: role of ovarian stem cells and epithelial-to-mesenchymal transition. J Cancer Sci Ther 2014; 6: 298-305. https:// doi.org/10.4172/1948-5956.1000284

[5] PARK EK, LEE JC, PARK JW, BANG SY, YI SA et al. Transcriptional repression of cancer stem cell marker CD133 by tumor suppressor p53. Cell Death Disease 2015; 6: e1964. https://doi.org/10.1038/cddis.2015.313.

[6] FENG Z, XU W, ZHANG CH, LIU M, WEN H. Inhibition of gamma-secretase in Notch1 signaling pathway a novel treatment for ovarian cancer. Oncotarget 2017; 5: 8215-8225. https://doi.org/10.18632/oncotarget.14152

[7] WEN Y, HOU Y, HUANG Z, CAI J, WANG Z. SOX2 is required to maintain cancer stem cells in ovarian cancer. Cancer Sci 2017; 108: 719-731. https://doi.org/10.1111/ cas. 13186 
[8] TOMAO F, PAPA A, STRUDEL M, ROSSI L, LO RUSSO $\mathrm{G}$ at al. Investigating molecular profiles of ovarian cancer: an update on cancer stem cells. J Cancer 2014; 5: 301-310. https://doi.org/10.7150/jca.8610

[9] ZHANG H, YANG Y, WANG Y, GAO X, WANG W et al. Relationship of tumor maker CA125 and ovarian tumor stem cells: preliminary identification. J Ovarian Res 2015; 8: 19. https://doi.org/10.1186/s13048-015-0132-8.

[10] YIN S, CHERYAN VT, XU L, RISHI AK, REDDY KB. Myc mediates cancer stem-like cells and EMT changes in triple negative breast cancers cells. PLoS One 2017; 12: e0183578. https://doi.org/10.1371/journal.pone.0183578

[11] GRINSTEIN RA, SHETZER Y, KAUFMAN T, ROTTER V. p53: the barrier to cancer stem cell formation. FEBS Letters 2014; 588: 2580-2589. https://doi.org/10.1016/j.febslet.2014.02.011

[12] LOUGHERY J, COX M, SMITH M, MEEK DW. Clinical role for 553-serine 15 phosphorylation in stimulating transactivation at p53-responsive. Nucleic Acids Res 2014; 47: 76667680. https://doi.org/10.1093/nar/gku501

[13] OLIVOS DJ, MAYO LD. Emerging non-canonical functions and regulation by p53: p53 and stemness. Int J Mol Sci 2016; 17. https://doi.org/10.3390/ijms17121982

[14] LIN T, LIN Y. p53 switches off pluripotency on differentiation. Stem Cell Res Ther 2017; 8: 44. https://doi.org/10.1186/ s13287-017-0498-1

[15] VELLETRI T, XIE N, WANG Y, HUANG Y, YANG Q et al. p53 functional abnormality in mesenchymal stem cells promotes osteosarcoma development. Cell Death Dis 2016; 7: e2015. https://doi.org/10.1038/cddis.2015.367

[16] LIU D, OU L, CLEMENSON GD, JR, CHAO C, LUTSKE $\mathrm{ME}$ et al. Puma is required for p53-induced depletion of adult stem cells. Nat Cell Biol 2010; 12: 993-998. https://doi. org/10.1038/ncb2100

[17] KAMINSKA I, BAR JK. The association between p53 protein phosphorylation at serine 15 , serine 20 and sensitivity of cells isolated from patients with ovarian cancer and cell lines to chemotherapy in in vitro study. Pharm Rep 2018; 70: 570-576. https://doi.org/10.1016/j.pharep.2017.12.004

[18] BENEDET JL, BENDER H, JONES H 3RD, NGAN HY, PECORELLI S. FIGO staging classifications and clinical practice gudelines in the management of gynecologic cancers. FIGO Committee on Gynecologic Oncology. Int J Gynaecol Obstet 2000; 70: 209-262. https://doi.org/10.1016/ S0020-7292(00)90001-8

[19] YOUNG RH, CLEMENT PB, SCULLY RE. Surface epithelial stromal tumors, pp 2319-2382. In Sternberg SS (Eds.). Diagnostic Surgical Pathology, 3rd Edition. Lippincott Williams \& Wilkins, Philadelphia 1999, p 2513. ISBN 0397587929.

[20] KHANDAKAR B, MATHUR SR, KUMAR L, KUMAR S, DATTA GUPTA $S$ et al. Tissue biomarkers prognostication of serous ovarian cancer following neoadjuvant chemotherapy. Biomed Res Int 2014; 2014: 401245. https://doi. org/10.1155/2014/401245

[21] IWANICKI MP, CHEN H-Y, IAVARONE C, ZERVANTONAKIS IK, MURANEN T et al. Mutant p53 regulates ovarian cancer transformed phenotypes trough autocrine matrix deposition. JCI Insight 2016; 1. https://doi.org/10.1172/jci. insight.86829
[22] ASLANI FS, MOMENI A, MOMTAHAN M, JAHROMI AM, DEHGHANIAN AR et al. Evaluation of p53 PTEN, and $\beta$-catenin immunoexpressions in primary ovarian epithelial tumors. Middle East J Cancer 2015; 6: 135-142.

[23] LIEW P-Y, FANG CH-Y, LEE Y-CH, LEE YC, CHEN CL et al. DEF6 expression in ovarian carcinoma correlates with poor patient survival. Diagn Pathol 2016; 11: 68. https://doi. org/10.1186/s13000-016-0518-y

[24] SEMCZUK A, GOGACZ M, SEMCZUK-SIKORA A, JOZWIK M, RECHBERGER T. The putative role of TP53 and p53 expression in borderline ovarian tumors - correlation with clinicopathological features and prognosis:a mini review. J Cancer 2017; 8: 2684-2691. https://doi.org/10.7150/ jca. 19691

[25] COLE AJ, DWIGHT T, GILL AJ, DICKSON KA, ZHU Y et al. Assessing mutant p53 in primary high- grade serous ovarian cancer using immunohistochemistry and massively parallel sequencing. Sci Rep 2016; 6: 26191-26203. https:// doi.org/10.1038/srep26191

[26] CHEN CH, WANG X, HUANG SH, WANG L, HAN L et al. Prognostic roles of Notch receptor mRNA expression in human ovarian cancer. Oncotarget 2017; 8: 32731-32740. https://doi.org/10.18632/oncotarget.16387

[27] ZHANG R, SHI H, REN F, LIU H, ZHANG M et al. Misregulation of polo-like protein kinase I, P53 and P21 in epithelial ovarian cancer suggests poor prognosis. Oncol Rep 2015; 33; 1235-1242. https://doi.org/10.3892/or.2015.3723

[28] BAR JK, SLOMSKA I, RABCZYNSKI J, NOGA L, GRYBOS M. Expression of p53 protein phosphorylated at serine 20 and serine 392 in malignant and benign ovarian neoplasms. Int J Gynecol Cancer 2009; 19: 1322-1328. https://doi. org/10.1111/IGC.0b013e3181b70465

[29] NGUYEN TA, MENENDEZ D, RESNICK MA, ANDERSON CW. Mutant TP53 posttranslational modifications: challenges and opportunities. Hum Mutat 2014; 35: 738-755. https://doi.org/10.1002/humu.22506

[30] LIANG J, YANG B, CAO Q, WU X. Association of vasculogenic mimicry formation and CD133 expression with poor prognosis in ovarian cancer. Gynecol Obstet Invest 2016; 81: 529-536. https://doi.org/10.1159/000445747

[31] ZHAO L, LI J, LIU M, ZHOU H, ZOU H et al. The clinicopathological parameters significance of CD133 and Nestin in epithelial ovarian cancer: a meta-analysis. Future Oncol 2017; 28: 2555-2570. https://doi.org/10.2217/fon-20170256

[32] WANG W, ZHANG Z, ZHAO Y, YUAN Z, YANG X et al. Enrichment and characterization of ovarian cancer stem cells and its potential clinical application. Int J Clin Exp Pathol 2016; 9: 10128-10138.

[33] ZHOU Q, CHEN A, SONG H, TAO J, YANG H et al. Prognostic value of cancer stem cell marker CD133 in ovarian cancer: a meta-analysis. Int J Clin Exp Med 2015; 8: 3080 3088.

[34] TONG T, ZHANG D-L. Detection of p53 and Bcl-2 expression in cutancous hemangioma through the quantum dot technique. Oncol Lett 2017; 13: 2937-2944. https://doi. org/10.3892/ol.2017.5856 
[35] MATSUDA K, KAWASAKI T, AKAZAWA Y, HASEGAWA $\mathrm{Y}, \mathrm{KONDO} \mathrm{H}$ et al. Expression pattern of p53-binding protein 1 as a new molecular indicator of genomic instability in bladder urothelial carcinoma. Sci Rep 2018; 8: 15477. https:// doi.org/10.1038/s41598-018-33761-9

[36] ALNIAIMI AN, DEMOREST-HAYES K, ALEXANDER VM, SEO S, YANG D et al. Increased Notch1 expression is associated with poor overall survival in patients with ovarian cancer. Int J Gynecol Cancer 2015; 25: 208-213. https://doi. org/10.1097/IGC.0000000000000359
[37] LI Y, CHEN K, LI L, LI R, ZHANG J et al. Overexpression of SOX2 is involved in paclitaxel resistance of ovarian cancer via the PI3K/Akt pathway. Tumour Biol 2015; 36: $9823-$ 9828. https://doi.org/10.1007/s13277-015-3561-5

[38] MIZUNO H, SPIKE BT, WAHL GM, LEVINE AJ. Inactivation of p53 in breast cancers correlated with stem cell transcriptional signatures. Proc Natl Acad Sci USA 2010; 107: 22745-22750. https://doi.org/10.1073/pnas.1017001108

[39] ARSIC N, GADEA G, LAGENQVIST EL, BUSSON M, CAHUZAC $\mathrm{N}$ et al. The $\mathrm{p} 53$ isoform $\triangle 133 \mathrm{p} 53 \beta$ promotes cancer stem cell potential. Stem Cell Res 2015; 4: 531-540. https://doi.org/10.1016/j.stemcr.2015.02.001 